\title{
CORRECTION
}

\section{Correction: Unanticipated rare adverse events and the surgeon startle response in ophthalmic surgery}

Angela Y. Chang, Grant A. Justin (D), Priya M. Mathews and James D. Auran (D)

(c) The Author(s), under exclusive licence to The Royal College of Ophthalmologists 2021

Eye (2022) 36:248; https://doi.org/10.1038/s41433-021-01742-4

Correction to: Eye https://doi.org/10.1038/s41433-021-01703-x, Published: 29 July 2021
Unfortunately, the spelling of the author's name Priya M. Mathews was incorrect.

The original article has been corrected. 